\title{
Impacto del uso de antimicrobianos en medicina veterinaria
}

\section{The antimicrobials use impact on veterinary medicine}

Fecha de recepción: 11 de febrero de 2017

Fecha de aprobación: 21 de mayo de 2017

\author{
Jenny Carolina Hernández-Barrera' \\ Maritza Angarita-Merchán² \\ Carlos Fernando Prada-Quiroga ${ }^{3}$
}

DOI: http://doi.org/10.19053/01228420.v14.n2.2017.7146

\section{Resumen}

Actualmente, la resistencia a los antimicrobianos es de gran interés a nivel mundial, debido a su impacto en la salud animal y humana y en la contaminación ambiental y a su costo económico. Se presentan diversos datos sobre la utilización de antibacterianos en la explotación pecuaria; sobre el porcentaje de antibacterianos detectados en el estiércol o en la orina animal, el tiempo que tarda en su degradación en el ambiente y la consecuente contaminación de varios ambientes, y sobre el impacto en la fauna salvaje. Se han descrito diferentes hipótesis sobre la resistencia a antimicrobianos, pero actualmente existen evidencias que indican que la utilización desmesurada de los antibacterianos en la medicina humana y la producción pecuaria es el factor determinante para el desarrollo de dicho mecanismo en los microorganismos.

Palabras clave: contaminación de alimentos; farmacorresistencia bacteriana; medicina veterinaria; profilaxis antibiótica. (Fuente: DeCS).

\begin{abstract}
Currently, antimicrobial resistance is of great interest worldwide, due to their impact on animal and human health, also on environmental contamination and their economic cost. We present some data about the use of multiple antibiotics in livestock farming; the percentage of antibiotics detected in animal manure or urine; their degradation time on environment and the resulting contamination of several environments and impact on wildlife.

11 M. Sc. Universidad de Boyacá (Tunja, Colombia). jenhernandez@uniboyaca.edu.co.

22 Mg. Universidad de Boyacá (Tunja, Colombia). mangarita@uniboyaca.edu.co. ORCID: 0000-0002-0220-2701.

33 Ph. D. Universidad de Boyacá (Tunja, Colombia). cfprada@uniboyaca.edu.co. ORCID: 0000-0001-5201-9502.
\end{abstract}


There have been described different hypotheses about antimicrobial resistance, but currently there are evidences which indicate that excessive use of antibiotics in human medical care and the livestock production are the determining factors for the development of these resistance, in the microorganisms, whose emergence could have a crucial role.

Keywords: antibiotic prophylaxis; bacterial drug resistance; food contamination; veterinary medicine (Source: $\mathrm{MeSH}$ ).

\section{Como citar:}

Hernández-Barrera JC., Angarita-Merchán M., Prada-Quiroga CF. Impacto del uso de antimicrobianos en medicina veterinaria. Rev. Cien. Agri. 2017; 14(2): 27-38. 


\section{Introducción}

La Organización Mundial de la Salud (OMS), la Organización de las Naciones Unidas para la Alimentación y la Agricultura (FAO) y la Organización Mundial de la Salud Animal (OIE) han mostrado especial interés en el preocupante problema de salud pública relacionado con el uso indiscriminado de antibacterianos, el surgimiento de cepas bacterianas resistentes a estos y su impacto en la salud humana y animal (1). Desde el inicio de la era de los antibacterianos, el incremento de la resistencia a estos ha sido descrito ampliamente. Durante varias décadas, la resistencia antimicrobiana se presenta como una amenaza creciente para el tratamiento eficaz de una amplia gama de infecciones causadas por bacterias, parásitos, virus y hongos (2); esta resistencia a antimicrobianos puede reducir la eficacia de los medicamentos para tratamiento infeccioso, como antibacterianos, antiparasitarios, antivirales y antifúngicos, tornando difícil el manejo de las infecciones en humanos y animales con este tipo de microorganismos resistentes (3). Inicialmente, el problema fue resuelto con la síntesis de nuevas substancias que fueron capaces de controlar los microorganismos con resistencia a antimicrobianos, pero después de la introducción en la clínica de cada nuevo medicamento, en un período variable de tiempo (algunos en pocos años) surgen cepas resistentes de bacterias (4).

El incremento de la resistencia a los antimicrobianos en distintos microorganismos se ha asociado a la combinación de diversos factores naturales o artificiales; uno de estos es la capacidad de intercambio genético existente entre las bacterias, el cual genera una discusión sobre la transferencia de genes de resistencia de microorganismos presentes en animales a los seres humanos; sin embargo, este proceso no ha sido fácil de comprobar. El uso indiscriminado de fármacos permitidos y prohibidos (moléculas promotoras de crecimiento), la aplicación de dosis inferiores a las necesarias para la prevención terapéutica de la enfermedad o, simplemente, para efectos "productivos", sin tener en cuenta el tiempo de retiro del animal, gracias a la consecución de alimentos altamente nutritivos, generan la aparición de residuos de estos medicamentos en los alimentos destinados para consumo humano, convirtiéndose en un factor de riesgo importante para la salud pública y un limitante para el desarrollo económico de cualquier país; es por ello que se han considerado por la FAO, la OIE y la OMS como procesos relevantes en el desarrollo de resistencia y objeto de vigilancia y control de los requisitos que se debe exigir en los alimentos destinados para consumo humano, principalmente de tipo exportación (1).

Por lo planteado, en esta revisión serán abordados los principales orígenes y consecuencias de la resistencia a antimicrobianos en bacterias asociadas a la salud animal, y su impacto en la salud de los humanos.

\section{Materiales y métodos}

Esta revisión de literatura se presenta como resultado de la ejecución del proyecto de investigación en genes de resistencia a antibacterianos en medicina veterinaria titulado "Detección de genes de resistencia a betalactámicos en aislamientos de Staphylococcus spp., asociados a mastitis bovina". Se realizó una búsqueda de publicaciones cuyo contenido mostrara el origen de la resistencia a antimicrobianos y su impacto en la medicina veterinaria. Para seleccionar los artículos fueron consultadas bases bibliográficas como Pubmed, a través del centro Nacional de Información Biotecnológica (NCBI), SciELO y Web de Ciencia. Fue estipulado un límite en las fechas de las publicaciones desde el año 2004 hasta el año 2014, utilizando los siguientes descriptores MESH: Drug Resistance Bacterial, Antibiotic Prophylaxis, Veterinary Medicine, Food Contamination. Los registros obtenidos oscilaron entre 28 y 4546 , tras la combinación de las diferentes palabras. Se seleccionaron aquellos documentos que indicaron sobre los aspectos microbiológicos y moleculares de la resistencia a antibacterianos en el ámbito veterinario y su impacto en salud pública. Fueron considerados artículos de investigación original y de revisión, disponibles en inglés o español. Además, fueron excluidos 
los trabajos que describieran modelos sin analizar los resultados, que no presentaran los datos del análisis o rigor metodológico, siguiendo las directrices internacionales de CONSORT, STARD, MOOSE, PRISMA y STROBE. Se incluyeron algunas publicaciones fuera del rango de tiempo mencionado, las cuales se emplearon como referentes históricos o teóricos.

\section{A. Uso de los antibacterianos en la medicina veterinaria}

Después del descubrimiento de los antibacterianos como principal tratamiento contra infecciones en humanos, se introdujeron progresivamente en medicina veterinaria. Animales como bovino s, caprinos, ovinos, porcinos, aves y peces, e ntre otros, tratados con antibacterianos para combatir ciertas enfermedades infecciosas, son util i zados para el consumo humano; por tanto, posiblemente transfieran a los humanos cepas bacteriana $s$ resistentes (5). Pero en las últimas décad a $s$, algunos antibacterianos han sido utilizado $s$, particularmente, con un propósito no terapéutico en la medicina veterinaria $(4,6)$; un ejem plo de ellos son los antibacterianos promotores $\mathrm{d}$ e I crecimiento (APC), que fueron propuestos p o $r$ primera vez a mediados de los años cincuenta, cuando se descubrió que medicamentos como la penicilina procaína y la tetraciclina, usados en pequeñas dosis (terapéuticas) en la alimentación, tienen la capacidad de incrementar el peso de aves, cerdos y bovinos de corte en una proporción cercana al 5 \% (7). Se supone que los APC actúan modificando cuantitativa y cualitativamente la microbiota intestinal, causando una diminución de microorganismos generadores de infecciones $(8,9)$; sin embargo, se han postulado otros mecanismos que explican el incremento del crecimiento mediado por los antibacterianos: (a) la reducción en la producción de metabolitos de crecimiento microbiano, (b) reducción de la utilización microbiana de nutrientes y (c) el aumento en la captación de nutrientes a través de la pared intestinal de animales a los que se les suministra antimicrobianos, llevando al aumento de la productividad (10). Por otro lado, en un análisis de 3000 raciones de alimentos para cerdos, en los Estados Unidos, cerca del 25 $\%$ contiene concentraciones más elevadas de los niveles recomendados (11).

Estudios realizados por Vela et al., en el 2014, reportan la presencia de residuos de betalactámicos (penicilina G), tetraciclinas (oxitetraciclina), macrólidos (eritromicina) y aminoglucósidos (estreptomicina ) en músculo bovino, sugiriendo la elección de métodos analíticos microbiológicos que apoyen la implementación del "Programa de Control de Residuos de Medicamentos Veterinarios" en Colombia, contribuyendo a garantizar la inocuidad alimentaria para proteger la salud de los consumidores (12).

La administración de diferentes antibacterianos en la industria ha aumentado drásticamente la resistencia a antimicrobianos en todo el mundo, especialmente en la Unión Europea o en países como Estados Unidos y China. Por ejemplo, en los Estados Unidos la cantidad de antibacterianos usados en 2004 fue 108 veces más que la utilizada en 1950; parte del incremento es debido a que los nivel es recomendados de APC en raciones de alimento de cerdos y aves aumentó de 4 ppm (para an tibacterianos de bajo espectro) y de 10 ppm (para los antibacterianos de amplio espectro), en 1950, a 200 ppm utilizadas actualmente (13). Según el reporte de la FDA (Food and Drug Administration of USA) generado en 2011, fueron utilizadas más de 13,5 toneladas de antibacterianos para uso veterinario en animales de producción, dentro de los que se destacan representantes de antimicrobianos como las tetraciclinas, penicilinas y amino g lucósidos (14), empleados en la actualidad.

Por otra parte, existen varios datos en la literatura sobre el uso inadecuado de antibacterianos en la industria pecuaria y su implicación negativa sobre la salud animal y humana en el continente americano y el mundo. Uno de los ejemplos para destacar es el de la acuicultura, que se caracteriza por la aplicación de antibacterianos, como método para el incremento de la producción; en Chile, por ejemplo, se ha reportado el uso de flumequina (una fluoroquinolona utilizada exclusivamente en acuicultura), la cual aumentó de 30 a 100 toneladas 
entre 1998 a 2002; este dato coincide con un incremento en la producción de salmón, que en el mismo período aumentó de $258 \mathrm{mil}$ a $494 \mathrm{mil}$ toneladas, ubicando a Chile como el segundo mayor productor de esta especie en cautiverio, después de Noruega (15).

De acuerdo con Kemper et al. (16), en Alemania los antibacterianos más utilizados en tratamientos terapéuticos y subterapéuticos en la industria pecuaria son: betalactámicos (penicilinas naturales y semisintéticas: amoxicilina, ampicilina, benzylpenicilina, cloxacilina, dicloxacilina, flucloxacilina, meticilina, mezlocilina, nafcilina, oxacilina, piperacilina, fenoxymetilcilina), macrólidos (azitromicina, claritromicina, clindamicina, eritromicina, roxithromicina, tilosina, vancomicina), sulfonamidas (sulfadimidina, sulfamethoxazole), trimetoprim, fluorquinolonas (ciprofloxacina, ofloxacina), tetraciclinas (clortetraciclina, doxiciclina, oxitetraciclina, tetraciclina), y el antibacteriano Poliéter (monensina); los antibacterianos que se encuentran subrayados son utilizados principalmente como medida terapéutica y subterapéutica en la industria bovina y porcícola (13).

De igual forma, en la última década los animales de compañía se han convertido en parte importante de la sociedad, por lo tanto, el mercado mundial de medicamentos para estos animales ha aumentado significativamente desde el inicio de los años noventa. Algunos países están incrementando la regulación del uso de los antibacterianos en animales de producción debido al posible efecto directo e indirecto en los humanos, pero esto no ocurre con los animales de compañía; algunos autores consideran que los antibacterianos usados para el cuidado de estos animales pueden constituir un importante porcentaje (cerca del 50 $\%$ ) del total aplicado a todos los animales, similar a lo reportado en Dinamarca (17).

En algunos países existen políticas para controlar el uso de antibacterianos, principalmente, con fines terapéuticos en animales. La mayoría de los países industrializados han generado políticas restrictivas en su uso; dichas políticas surgieron de estudios que presentaban a la comunidad científica la relación tangible entre el uso indiscriminado y el origen de la resistencia a antimicrobianos (7); por ejemplo, al final de los años sesenta, surgió la preocupación sobre el aumento de resistencia, principalmente bacteriana, y su posible relación con el uso de APC $(16,17)$. Según la reglamentación vigente del Instituto Colombiano Agropecuario (ICA), se recomienda no utilizar sustancias antimicrobianas como promotores de crecimiento, cuando ellas se empleen como agentes terapéuticos en medicina humana o medicina veterinaria, de acuerdo con la resolución N ${ }^{\circ} 002341$ (23 de agosto de 2007), por la cual se reglamentan las condiciones sanitarias y de inocuidad en la producción primaria de ganado bovino y bufalino destinado al sacrificio para consumo humano; sin embargo, solo hay unos pocos antibacterianos registrados como APC, lo que dificulta su inspección.

En 1960 fue publicado el informe británico Swann, que alertó sobre el riesgo potencial de selección de bacterias resistentes en animales, las cuales podrían ser ingeridas por los seres humanos (18); el informe recomiendó que no fueran usados los antibacterianos como promotores de crecimiento, por un posible efecto de resistencia cruzada. En 1970, la ex-Comisión Europea (CEE) publicó la directiva CEE 70/524, relacionada con los aditivos en la alimentación animal, donde se señala que solo pueden ser empleados como promotores los antibacterianos que tengan un efecto comprobado sobre el crecimiento de los animales, que fueran activos contra las bacterias Gram-positivas y que no promuevan la absorción intestinal, para evitar residuos en la carne (6); de esta forma, fue prohibido en Europa el uso de tetraciclinas o betalactámicos como APC en la alimentación animal (19).

En Colombia debe prestarse especial interés a la reglamentación sobre las condiciones sanitarias y de inocuidad en la producción primaria, como es el caso de los documentos del Consejo Nacional de Política Económica y Social (CONPES), en los que se consolidan las políticas sanitarias y de inocuidad para las diferentes cadenas: carne bovina y leche (CONPES 3376), y la resolución 1382 de 02 de mayo de 2013, en donde se establecen los límites máximo de residuos de medicamentos en alimentos 
de origen animal destinados para consumo humano; dichas reglamentaciones posibilitan el acceso a mercados internacionales, dentro de cuyas exigencias para la comercialización de este tipo de productos se encuentra evitar al máximo el uso de aditivos como antimicrobianos.

\section{B. Contaminación ambiental por antibacterianos}

Además del uso directo y exagerado de los antibacterianos en animales, existen evidencias científicas de contaminación ambiental con residuos de antibacterianos. Una de las principales fuentes de contaminación con estos productos son las excretas de los animales, debido a que estos no utilizan todos los antibacterianos presentes en la alimentación o en el tratamiento de las infecciones, y un alto porcentaje son excretados. Estudios realizados en diferentes especies animales de producción demuestran que, dependiendo de la molécula, algunos antibacterianos pueden ser excretados intactos entre el $30 \%$ y el $90 \%$ (20). Algunos metabolitos son más eficaces que los compuestos de origen; por ejemplo, los conjugados acéticos de sulfonamidas pueden volver a sus compuestos de origen durante el almacenamiento del estiércol (21). Un estudio realizado en 126 muestras de estiércol de cerdos en Shandong (China) determinó la presencia del $85 \%-97 \%$ de tetraciclinas, $52 \%$ de sulfonamidas y $5 \%$ de macrólidos intactos (22); resultados semejantes fueron reportados en otros estados de China (23) y en el Japón (24). De igual forma, las tetraciclinas (especialmente oxitetraciclina y clortetraciclina) han sido detectadas en todo el mundo en muestras de lagos o de estiércol de animales de granja (25). Normalmente, las concentraciones de antibacterianos contenidos en este material oscilan entre $1-10 \mathrm{mg} \cdot \mathrm{kg}^{-1} \mathrm{o} \mathrm{L}^{-1}$, pero pueden Ilegar a niveles $\square 200 \mathrm{mg} \cdot \mathrm{kg}^{-1} \mathrm{o} \mathrm{L} \mathrm{L}^{-1}$ (19). En este caso, han sido detectadas concentraciones de hasta $216 \mathrm{mg} \cdot \mathrm{kg}^{-1}$ en muestras de estiércol de cerdos, bovinos y aves/pavos (13). Varios estudios han confirmado que los antibacterianos usados en animales de producción están presentes, además, en suelo, en aguas superficiales y subterráneas adyacentes a la producción pecuaria (26).

La presencia de antibacterianos en el estiércol varía dependiendo de la clase; el análisis se encuentra resumido en la Tabla I.

Tabla I. Concentración de antibacterianos en estiércol de animales de granja. Adaptada de (12).

\begin{tabular}{|c|c|c|c|c|}
\hline Antibacteriano & Tipo de estiércol & Concentración & $\begin{array}{c}\text { Vida media en el } \\
\text { ambiente }\end{array}$ & Referencia \\
\hline Oxitetraciclina & \multirow{2}{*}{$\begin{array}{l}\text { Estiércol de cerdos, } \\
\text { pollos y pavos }\end{array}$} & $136 \mathrm{mg} \cdot \mathrm{L}^{-1}$ & 21-35 días & \multirow[t]{2}{*}{$(27)$} \\
\hline Clortetraciclina & & $46 \mathrm{mg} \cdot \mathrm{L}^{-1}$ & 100 días & \\
\hline Tetraciclina & \multirow{5}{*}{ Estiércol de cerdos } & $98 \mathrm{~g} \cdot \mathrm{L}^{-1}$ & 100 días & \multirow[t]{5}{*}{ (23) } \\
\hline Oxitetraciclina & & $354 \mathrm{mg} \cdot \mathrm{L}^{-1}$ & 21-35 días & \\
\hline Clortetraciclina & & $139 \mathrm{mg} \cdot \mathrm{L}^{-1}$ & 100 días & \\
\hline Doxiciclina & & $37 \mathrm{mg} \cdot \mathrm{L}^{-1}$ & 100 días & \\
\hline Sulfadiazina & & $7,1 \mathrm{mg} \cdot \mathrm{L}^{-1}$ & 8-30 días & \\
\hline Tetraciclina & \multirow[t]{2}{*}{ Estiércol de cerdos } & $30 \mathrm{mg} \cdot \mathrm{kg}^{-1}$ & 100 días & \multirow[t]{2}{*}{$(28)$} \\
\hline Sulfonamidas & & $2 \mathrm{mg} \cdot \mathrm{kg}^{-1}$ & 8 - 30 días & \\
\hline Tilosina & \multirow{2}{*}{$\begin{array}{l}\text { Estiércol fresco de } \\
\text { becerro }\end{array}$} & $0,11 \mathrm{mg} \cdot \mathrm{kg}^{-1}$ & 2-21 días & \multirow[t]{2}{*}{$(29)$} \\
\hline Oxitetraciclina & & $10 \mathrm{mg} \cdot \mathrm{kg}^{-1}$ & 21-35 días & \\
\hline Clortetraciclina & \multirow{3}{*}{$\begin{array}{l}\text { Estiércol almacenado } \\
\text { de bovino }\end{array}$} & $6,6 \mathrm{mg} \cdot \mathrm{kg}^{-1}$ & 100 días & \multirow[t]{3}{*}{$(30)$} \\
\hline Monensina & & $120 \mathrm{mg} \cdot \mathrm{kg}^{-1}$ & 28 días & \\
\hline Tilosina & & $8,1 \mathrm{mg} \cdot \mathrm{kg}^{-1}$ & 45 días & \\
\hline
\end{tabular}




\begin{tabular}{|l|l|l|c|l|}
\hline Antibacteriano & \multicolumn{1}{|c|}{ Tipo de estiércol } & \multicolumn{1}{|c|}{ Concentración } & $\begin{array}{c}\text { Vida media en el } \\
\text { ambiente }\end{array}$ & Referencia \\
\hline Oxitetraciclina & Estiércol de vaca & $0,5-200 \mathrm{mg} \cdot \mathrm{L}^{-1}$ & $21-35$ días & $(31)$ \\
\hline Clortetraciclina & Estiércol de cerdos & $764,4 \mathrm{mg} \cdot \mathrm{L}^{-1}$ & 100 días & $(24)$ \\
\hline Clortetraciclina & $\begin{array}{l}\text { Estiércol almacenado } \\
\text { de cerdos }\end{array}$ & $1 \mathrm{mg} \cdot \mathrm{L}^{-1}$ & 100 días & \multirow{2}{*}{$(32)$} \\
\cline { 1 - 4 } Oxitetraciclina & Lagos de piscicultura & $0,41 \mathrm{mg} \cdot \mathrm{L}^{-1}$ & $21-35$ días & \\
\hline
\end{tabular}

Los anteriores reportes demuestran el impacto generado por la difícil biodegradación de la mayoría de los antibacterianos, acumulándose y dispersándose a lo largo de las explotaciones pecuarias.

Cabe destacar que la aplicación de estiércol es una práctica común en muchas partes del mundo, utilizándose principalmente como un fertilizante eficiente y económico; en Estados Unidos, este material es aplicado con frecuencia, incluso durante el invierno sobre la nieve (20). Un estudio realizado por Vázquez-Vázquez et al. muestra que además de proveer de materia orgánica y nutriente de importancia agrícola, como el nitrógeno, los antibacterianos presentes en el estiércol pueden prevenir, controlar e influenciar el grado de ataque de patógenos en plantas (33).

En 2006 fue reportado que las operaciones pecuarias en los Estados Unidos produjeron cerca de 133 millones de toneladas de estiércol (34), de las cuales la cría de 800 mil cerdos podría producir más de 1,6 millones de toneladas de residuos por año (35). De igual forma, se estima que el ganado vacuno en los Estados Unidos genera entre 1,9 y 14,2 millones de toneladas de estiércol/día (36). Por lo tanto, la producción de estiércol depende del tipo de explotación pecuaria. En este caso, se estima un volumen promedio de estiércol fresco generado por cada día de $0,102 \mathrm{~kg} /$ pollos de engorde, 0.270 kg/pavo, $4.7 \mathrm{~kg} / \mathrm{cerdos}$ de engorde, $22 \mathrm{~kg} /$ ganado de engorde, $38 \mathrm{~kg}$ seco/vaca y $68 \mathrm{~kg} /$ vacas lecheras (37). Actualmente, existen varios mecanismos que regulan la utilización o eliminación del estiércol procedente de las operaciones de alimentación de animales en confinamiento (CAFO, sigla en inglés). Por ejemplo, la EPA (Environmental Protection Agency of USA) es la responsable por monitorear y certificar las descargas de estos residuos de producción a los cuerpos de agua (superficial y subterránea) producidos por las CAFO en los Estados Unidos (38).

En Suramérica existen pocos datos confiables sobre la producción de estiércol animal; sin embargo, se conoce que en Argentina el ganado en confinamiento produce entre $350.000 \mathrm{y}$ $600.000 \mathrm{mg}^{-a n ̃ o} \mathrm{o}^{-1}$ de estiércol seco (39). A pesar de su impacto, en Suramérica no existen agencias estatales o leyes que regulen estrictamente el vertimiento de estiércol de producción animal al ambiente; no obstante, existen iniciativas de algunos países en la búsqueda del control y prevención de la contaminación de cuerpos de aguas (especialmente de agua potable); por ejemplo, el Decreto 3930 de 2010 del Ministerio de Medio Ambiente de Colombia, o el Decreto 2009/60 en Argentina, la Ley 19300 en Chile, la Ley General de Equilibrio Ecológico y Protección Ambiental en México, entre otros (40).

Adicionalmente, ese tipo de fertilizante natural tiene mucho interés en la agricultura debido a la creciente demanda por alimentos orgánicos que desde hace dos décadas está en fase de crecimiento y ha aumentado con mayor intensidad en los últimos años (41). Chee-Sanford et al. (42) sugirieron que la aplicación continua de estiércol llevará a una tierra saturada de antibacterianos y, así, el agua subterránea se puede tornar en una fuente potencial de antibacterianos en la cadena alimentaria. Otros autores, como Daughton C. et al. (2008), Angenent L. et al. (2008), Pan X. et al. (2011), Chen Y. et al. (2012) y Daniel I. et al. (2014), han demostrado que las concentraciones de sulfonamida-sulfametazina y de otros antibacterianos son mayores en un lago rodeado por operaciones pecuarias intensivas que 
en los afluentes de estaciones de tratamiento de aguas residuales en la misma área (43). Por lo tanto, la utilización de estiércol como fertilizante se puede convertir en una importante fuente de contaminación de antibacterianos en suelos, ríos, lagos y otros ambientes.

Las explotaciones acuícolas son otras fuentes importantes de contaminación por antibacterianos. En la acuicultura moderna, las cajas y jaulas flotantes se encuentran, por lo general, en ambientes acuáticos, ya sean naturales o artificiales, habitadas por peces nativos y mariscos usados también para consumo humano. Algunas de estas especies se nutren de los alimentos no consumidos o de las heces de los peces cultivados que se acumulan debajo de las jaulas (44); de esta forma, diferentes especies nativas de peces y moluscos pueden ser involuntariamente expuestas a varios antibacterianos, con la capacidad de transmitirlos al tracto digestivo de sus respectivos consumidores (44).

\section{Biodegradación}

Existen diferentes estudios sobre la biodegradación de distintos antibacterianos después de su utilización. Algunos autores han demostrado que la mayoría de los antibacterianos tiene una vida media (t1/2) prolongada de 68 a 137 días, aproximadamente, durante el almacenamiento del estiércol o en el suelo. Algunos antibacterianos pueden tener una vida media de un día, como en el caso de la clindamicina o la claritromicina, o entre 37 y más de 77 días, en el caso de las tetraciclinas o doxiciclinas en el estiércol de diferentes animales de granja (45). Sin embargo, la vida media de los antibacterianos en el estiércol también depende del origen animal; por ejemplo, antibacterianos como las tetraciclinas, detectados en el estiércol de la vaca lechera, tienen una vida media de 17,7 días, más prolongada que en el estiércol del caballo, con 8,4 días (46).

Diferentes tipos de procesos biológicos afectan la persistencia de los antibacterianos; es así como la vida media de la clortetraciclina se incrementa en el siguiente orden: compostaje> estiércol almacenado>suelo. Se ha observado que la vida media para la degradación primaria de los antibacterianos en suspensiones aeróbicas de suelo-estiércol varia de 3.3 a 8.1 días para la tilosina, de 5.8-8.8 días para olaquindox y de 13.1-26.9 días para el metronidazol (47). Hektoen et al. (48) demostraron que los antibacterianos enterrados en sedimentos superficiales de lagos $(1-7 \mathrm{~cm})$ pueden tener una vida media de más de 300 días; esto significa que los antibacterianos pueden acumularse en el medio acuático con niveles peligrosos que pueden afectar comunidades bentónicas, las cuales se pueden perpetuar a través de la cadena trófica.

Existe un debate sobre la necesidad de obtener más análisis para otros antibacterianos de diferentes clases, con el fin de apoyar esta hipótesis y realizar más investigaciones para esclarecer las vías de degradación e identificación de metabolitos intermedios producidos en este proceso. Adicionalmente, las pobres recuperaciones de antibacterianos asociados con las técnicas bioquímicas de extracción utilizadas (31 \%-83 $\%$ de recuperación) y efectos de la matriz (50\% $-90 \%$ ) pueden explicar en parte la variación en los resultados de diversos estudios (13). De la misma forma, la falta de estudios en países en vía de desarrollo, como en América Latina, propone un panorama parcial de lo que está ocurriendo con la utilización, degradación y presencia de antibacterianos en la naturaleza.

\section{Efecto de los antibacterianos en el ambiente y su impacto en la salud pública}

Dentro de las principales preocupaciones por el efecto que los antibacterianos puedan tener sobre la salud humana se encuentra la posibilidad de que niveles tróficos enteros de bacterias podrían ser eliminados en ecosistemas contaminados con antibacterianos, o que bacterias con resistencia a antimicrobianos puedan florecer en la cadena alimenticia; por lo tanto, comunidades bacterianas y fúngicas del suelo, con una importante tarea en el ciclo de nutrientes, podrían ser alteradas o extinguidas (35).

Existen diferentes ejemplos en la literatura de cómo los antibacterianos afectan los ecosistemas; ejemplo 
de ello es el ácido oxolínico, normalmente utilizado en la industria acuícola, el cual, demostrado por pruebas de laboratorio, es extremamente tóxico para Daphnia magna, un crustáceo de agua dulce de gran importancia trófica, cuya capacidad reproductiva es completamente destruida por este antibacteriano (49). Por otro lado, la creciente preocupación por la muerte en masa de abejas en todo el mundo ha llevado a realizar diferentes estudios que demuestren el origen del problema; un equipo de investigadores de la Universidad de Yale indicó que los antibacterianos alteran drásticamente el microbioma de las abejas (y en general en los Hymenoptera), esencial para su nutrición y desarrollo (50), afectando no solo la producción de miel, sino también la polinización de centenas de especies de plantas, incluyendo especies de importancia económica.

\section{E. Posibles mecanismos de la transmisión de la resistencia a antimicrobianos en bacterias}

Desde el inicio de la era del antibacteriano, la incidencia de la resistencia a esas substancias ha sido descrita. Durante varias décadas, la resistencia a antimicrobianos se ha convertido en una amenaza creciente para el tratamiento eficaz de una gama cada vez mayor de infecciones causadas por bacterias e, incluso, por otros microorganismos. Los genes que codifican para la resistencia a los antibacterianos fluyen desde y para bacterias Gram-negativas y Gram-positivas, que habitan nichos diferentes (4). La "hipótesis del reservorio" presupone que determinada concentración-límite de antibacterianos es necesaria para inducir (o seleccionar) y después mantener la resistencia; asumiendo que los genes mutantes responsables de la resistencia son fenotípicamente neutros, pero que genera alguna incompetitividad por las bacterias en la ausencia del fármaco (4). Las pequeñas dosis de antibacterianos usadas como promotores de crecimiento podrían sustentar la hipótesis del reservorio y el surgimiento generalizado de la resistencia a antimicrobianos en diferentes animales.

Igualmente, el tracto gastrointestinal en los animales y la piel en los humanos han sido considerados los lugares predilectos de la transferencia de genes de resistencia; pero hoy otros nichos comienzan a ser considerados importantes en este aspecto; es así como el intestino de animales salvajes (especialmente roedores), de animales de compañía y de peces, especialmente en explotaciones pecuarias para la producción de estos, representan lugares donde el fenómeno puede ocurrir en gran escala (4). En ciertos lugares, como la tierra o pequeños cuerpos de agua $y$, especialmente, en áreas donde se descarga o almacena materia fecal, se podría presentar también este fenómeno. Estudios metagenómicos recientes demuestran que en el estiércol fresco de vaca existe alta frecuencia de genes de resistencia a antibacterianos con potencial dispersivo en la agricultura y en los animales, con un potencial impacto sobre la salud humana (51). Es por ello que el reservorio de bacterias resistentes en animales de producción implica un riesgo potencial para la transferencia de genes de resistencia en animales de consumo para los seres humanos. Adicionalmente, se ha demostrado que las bacterias con resistencia a antimicrobianos pueden ser transferidas directamente de animales a humanos, las cuales podrían infectar el suelo, alimentos y aguas a través de la aplicación del estiércol en el campo agrícola $(52,53)$. En resumen, algunas infecciones en seres humanos, causada por bacterias resistentes provenientes de animales, se han convertido en un factor de riesgo para salud pública.

\section{Conclusiones}

A lo largo de los años se ha tornado evidente que la utilización de antibacterianos como promotores de crecimiento en animales ha tenido un impacto negativo sobre el ambiente y la cadena trófica, generado a partir de la presencia de esos antimicrobianos, o genes de resistencia, en los residuos biológicos o productos de dichos animales. Un factor de riesgo potencial para la resistencia a los antibacterianos en humanos puede ser causado por la transferencia de bacterias que contengan genes de resistencia, presentes en alimentos de origen animal.

Algunos antibacterianos son secretados a través de fluidos animales, como orina y estiércol, en 
concentraciones significativas, los cuales son difícilmente biodegradados en el ambiente, como suelo, agua y cadenas tróficas en general. Algunas de las bacterias encontradas en el estiércol pueden ser perjudiciales para los seres humanos, $y$, adicionalmente, si estas son resistentes a los antibacterianos podrían causar graves problemas de salud pública.

El uso de estiércol de algunos animales en el sector agroindustrial puede ser un factor de riesgo para la salud humana, ante la necesidad de tratamientos de enfermedades infecciosas, por la transferencia de microorganismos (principalmente bacterias) con genes de resistencia a antibacterianos.

Es importante considerar el control y la regulación de uso de antibacterianos en medicina veterinaria, en especial aquellos empelados como APC.

El problema de la resistencia antimicrobiana se ha extendido a todos los sectores agropecuarios e industriales, los cuales han visto disminuir los productos que cumplen con los requisitos necesarios para ser avalados como productos alimenticios de calidad y aptos para consumo humano, afectando a países cuya economía gira alrededor de estos sectores.

La implementación de buenas prácticas y el cumplimiento de políticas sanitarias y de inocuidad de los alimentos podrían minimizar el uso indiscriminado de antibacterianos, mitigando el problema de la resistencia antimicrobiana en la práctica veterinaria y en la salud humana.

\section{Referencias}

(1) FAO/OIE/WHO. Joint FAO/OIE/WHO Expert Workshop on Non-Human Antimicrobial Usage and Antimicrobial Resistance: scientific assessment: Geneva, Diciembre 1-5, 2004.

(2) Cires Pujol M. La resistencia a los antimicrobianos, un problema mundial. Rev Cubana Med Gen Integr. 2002; 18(2): 165-8.

(3) Pantozzi F., Moredo F., Vigo G., Giacoboni G. Resistencia a los antimicrobianos en bacterias indicadoras y zoonóticas aisladas de animales domésticos en Argentina. Rev Argent Microbiol. 2010; 42(1): 49-52.

(4) Errecalde JO. Uso de antimicrobianos en animales de consumo: incidencia del desarrollo de resistencias en salud pública: FAO; 2004.
(5) Gundogan N., Citak S., Yucel N., Devren A. A note on the incidence and antibiotic resistance of Staphylococcus aureus isolated from meat and chicken samples. Meat Sci. 2005; 69(4): 807-10. DOI: http://doi.org/10.1016/j.meatsci.2004.10.011.

(6) Grande BC., Falcón MG., Gándara JS. El uso de los antibacterianos en la alimentación animal: perspectiva actual. Cyta-J Food. 2000; 3(1): 39-47.

(7) Seal BS, Lillehoj HS, Donovan DM, Gay CG. Alternatives to antibiotics: a symposium on the challenges and solutions for animal production. Anim Health Res Rev. 2013; 14(01): 78-87. DOI: http://doi.org/10.1017/S1466252313000030.

(8) Huyghebaert G., Ducatelle R., Van Immerseel F. An update on alternatives to antimicrobial growth promoters for broilers. Vet J., 2011; 187 (2): 182-188. DOI: http://doi.org/10.1016/j.tvjl.2010.03.003.

(9) Panda K., Rao SR., Raju M. Natural growth promoters have potential in poltry feeding systems. Animal Feed Science and Technology. 2006; 10(8): 23-25.

(10) Gaskins H., Collier C., Anderson D. Antibiotics as growth promotants: mode of action. Anim Biotechnol. 2002; 13(1): 29-42. DOI: http://doi.org/10.1081/ABIO-120005768.

(11) Dewey CE., Cox BD., Straw BE., Bush EJ., Hurd HS. Associations between off-label feed additives and farm size, veterinary consultant use, and animal age. Prev Vet Med., 1997; 31(1): 133-146. DOI: http://doi.org/10.1016/S0167-5877(96)01077-X.

(12) Vela-Perdomo KL., et al. Evaluación preliminar del bioensayo para la detección de antimicrobianos en músculo bovino. Vitae. 2014; 21(3): 178-190.

(13) Massé DI., Saady NM., Gilbert Y. Potential of Biological Processes to Eliminate Antibiotics in Livestock Manure: An Overview. Animals (Basel). 2014; 4(2): 146-163. DOI: http://doi.org/10.3390/ ani4020146.

(14) Food, Administration D. Summary Report on Antimicrobials Sold or Distributed for Use in Food-Producing Animals. FDA. 2011.

(15) Soto D. Evaluation of salmon farming effects on marine systems in the inner seas of sothern Chile: a largelscale mensurative experiment. J. Appl Ichthyol. 2004; 20(6): 493-501. DOI: http://doi. org/10.1111/j.1439-0426.2004.00602.x.

(16) Kemper N., Färber H., Skutlarek D., Krieter J. Analysis of antibiotic residues in liquid manure and leachate of dairy farms in Northern Germany. Agric. Water Manage. 2008; 95(11): 1288-1292. DOI: http://doi.org/10.1016/j.agwat.2008.05.008.

(17) Prescott JF. Antimicrobial use in food and companion animals. Anim Health Res Rev. 2008; 9(2): 127-133. DOI: http://doi. org/10.1017/S1466252308001473.

(18) Swann M., Baxter K., Field H. Report of the joint committee on the use of antibiotics in animal husbandry and veterinary medicine. Her Majesty's Stationary Office, London. 1969.

(19) Castanon J. History of the use of antibiotic as growth promoters in European poltry feeds. J. Anim Sci. 2007; 86 (11): 2466-2471.

(20) Kumar K., Gupta S., Baidoo S., Chander Y., Rosen C. Antibiotic uptake by plants from soil fertilized with animal manure. J. Environ Qual. 2005; 34(6): 2082-2085. DOI: http://doi.org/10.2134/ jeq2005.0026.

(21) Boxall A., Blackwell P., Cavallo R., Kay P., Tolls J. The sorption and transport of a sulphonamide antibiotic in soil systems. Toxi-

36 - Revista Ciencia y Agricultura (Rev. Cien. Agri.) Vol. 14 (2). ISSN 0122-8420. Julio - Diciembre 2017, pp. 27-38. Tunja (Boyacá) - Colombia. 
col. Lett., 2002; 131(1): 19-28. DOI: http://doi.org/10.1016/S03784274(02)00063-2.

(22) Pan X., Qiang Z., Ben W., Chen M. Residual veterinary antibiotics in swine manure from concentrated animal feeding operations in Shandong Province, China. Chemosphere. 2011; 84 (5): 695-700. DOI: http://doi.org/10.1016/j.chemosphere.2011.03.022.

(23) Chen Y., Zhang H., Luo Y., Song J. Occurrence and assessment of veterinary antibiotics in swine manures: A case study in East China. Chinese Science Bulletin. 2012; 57(6): 606-614. DOI: http://doi. org/10.1007/s11434-011-4830-3.

(24) Motoyama M, Nakagawa S, Tanoe R, Sato Y, Nomiyama K, Shinohara R. Residues of pharmaceutical products in recycled organic manure produced from sewage sludge and solid waste from livestock and relationship to their fermentation level. Chemosphere, 2011; 84(4): 432-8. DOI: http://doi.org/10.1016/j.chemosphere.2011.03.048.

(25) Aust M-O., Godlinski F., Travis GR., Hao X., McAllister TA., Leinweber $\mathrm{P}$. Distribution of sulfamethazine, chlortetracycline and tylosin in manure and soil of Canadian feedlots after subtherapeutic use in cattle. Environ Pollut. 2008; 156(3): 1243-1251. DOI: http:// doi.org/10.1016/j.envpol.2008.03.011.

(26) Huang C-H., Renew JE., Smeby KL., Pinkston K., Sedlak DL. Assessment of potential antibiotic contaminants in water and preliminary occurrence analysis. J. Contemp Water Res Educ. 2011; 120(1): 4.

(27) Martínez-Carballo E., González-Barreiro C., Scharf S., Gans O. Environmental monitoring study of selected veterinary antibiotics in animal manure and soils in Austria. Environ Pollut. 2007; 148(2): 570-579. DOI: http://doi.org/10.1016/j.envpol.2006.11.035.

(28) Jacobsen AM., Halling-Sørensen B. Multi-component analysis of tetracyclines, sulfonamides and tylosin in swine manure by liquid chromatography-tandem mass spectrometry. Anal. Biochem, 2006; 384(5): 1164-1174. DOI: http://doi.org/10.1007/s00216005-0261-9.

(29) De Liguoro M., Cibin V., Capolongo F., Halling-Sørensen B., Montesissa C. Use of oxytetracycline and tylosin in intensive calf farming: evaluation of transfer to manure and soil. Chemosphere. 2003; 52(1): 203-212. DOI: http://doi.org/10.1016/S00456535(03)00284-4.

(30) Dolliver HA., Gupta SC. Antibiotic losses from unprotected manure stockpiles. J. Environ Qual. 2008; 37(3): 1238-1244. DOI: http:// doi.org/10.2134/jeq2007.0391.

(31) Ince B., Coban H., Turker G., Ertekin E., Ince O. Effect of oxytetracycline on biogas production and active microbial populations during batch anaerobic digestion of cow manure. Bioprocess Biosyst Eng. 2013; 36(5): 541-546. DOI: http://doi.org/10.1007/s00449012-0809-y.

(32) Campagnolo ER., Johnson KR., Karpati A., Rubin CS., Kolpin DW., Meyer MT. Antimicrobial residues in animal waste and water resorces proximal to large-scale swine and poltry feeding operations. Sci. Total Environ. 2002; 299(1): 89-95. DOI: http://doi.org/10.1016/ S0048-9697(02)00233-4.

(33) Vázquez-Vázquez C., García-Hernández JL., Salazar-Sosa E., López-Martínez JD., Valdez-Cepeda RD., Orona-Castillo I. Aplicación de estiércol solarizado al suelo y la producción de chile jalapeño (Capsicum annuum L.). Rev. Chapingo Ser. Hortic. 2011; 17(1): 69-74. DOI: http://doi.org/10.5154/r.rchsh.2011.17.040.

(34) Burkholder J., Libra B., Weyer P., Heathcote S., Kolpin D., Thorne PS. Impacts of waste from concentrated animal feeding operations on water quality. Environ Health Perspect., 2007; 115(2): 308. http://doi.org/10.1289/ehp.8839.

(35) Hribar C., Schultz M. Understanding concentrated animal feeding operations and their impact on communities. Bowling Green, $\mathrm{OH}$. Nalboh. February. 2010; 18: 2013.

(36) NASS. Cattle on feed. USDA economics, statistics and market information system Cornell University, Ithaca, NY, and USDA, Washington, DC. 2010.

(37) Nennich T., Harrison J., Van Wieringen L., Meyer D., Heinrichs A., Weiss W. Prediction of manure and nutrient excretion from dairy cattle. J. Dairy Sci., 2005; 88(10): 3721-3733. DOI: http://doi. org/10.3168/jds.S0022-0302(05)73058-7.

(38) EPA. Animal Feeding Operations-Laws, Regulations, Policies and Guidance. Usepa. 2010: pp: 15.

(39) Bolton A., Studdert G., Echeverría H. Utilización de estiércol de animales en confinamiento como fuente de recurso para la agricultura. Rev Arg Prod An. 2004; 24(1-2): 53-74.

(40) Pinos-Rodríguez JM., García-López JC., Peña-Avelino LY., Rendón-Huerta JA., González-González C., Tristán-Patiño F. Impactos y regulaciones ambientales del estiércol generado por los sistemas ganaderos de algunos países de América. Agrociencia. 2012; 46(4): 359-370.

(41) Willer H., Kilcher L. The World of Organic Agriculture: Statistics and Emerging Trends 2011: Bonn: International Federation of Organic Agriculture Movements (IFOAM). FiBL. 2011.

(42) Chee-Sanford JC., Aminov RI., Krapac I., Garrigues-Jeanjean N., Mackie RI. Occurrence and diversity of tetracycline resistance genes in lagoons and grondwater underlying two swine production facilities. Appl Environ Microbiol. 2001; 67(4): 1494-1502. DOI: http://doi.org/10.1128/AEM.67.4.1494-1502.2001.

(43) Daughton CG., Jones-Lepp TL. Pharmaceuticals and personal care products in the environment: scientific and regulatory issues. American Chemical Society. J. Am. Chem. Soc. 2001.

(44) Buschmann AH., Cabello F., Yong K., Carvajal J., Varela DA., Henríquez L. Salmon aquaculture and coastal ecosystem health in Chile: analysis of regulations, environmental impacts and bioremediation systems. Ocean \& Coastal Management. 2009; 52(5): 243249. DOI: http://doi.org/10.1016/j.ocecoaman.2009.03.002.

(45) Chenxi W., Spongberg AL., Witter JD. Determination of the persistence of pharmaceuticals in biosolids using liquid-chromatography tandem mass spectrometry. Chemosphere. 2008; 73(4): 511-518. DOI: http://doi.org/10.1016/j.chemosphere.2008.06.026.

(46) Storteboom HN., Kim S-C., Doesken KC., Carlson KH., Davis JG., Pruden A. Response of antibiotics and resistance genes to high-intensity and low-intensity manure management. J. Environ Qual. 2007; 36(6): 1695-1703. DOI: http://doi.org/10.2134/ jeq2007.0006.

(47) Ingerslev F., Halling-Sørensen B. Biodegradability of metronidazole, olaquindox, and tylosin and formation of tylosin degradation products in aerobic soil-manure slurries. Ecotoxicol Environ Saf. 2001; 48(3): 311-320. DOI: http://doi.org/10.1006/eesa.2000.2026. 
(48) Hektoen H., Berge JA., Hormazabal V., Yndestad M. Persistence of antibacterial agents in marine sediments. Aquaculture. 1995; 133(3): 175-184. DOI: http://doi.org/10.1016/0044-8486(94)00310-K.

(49) Wollenberger L., Halling-Sørensen B., Kusk KO. Acute and chronic toxicity of veterinary antibiotics to Daphnia magna. Chemosphere. 2000; 40(7): 723-730. DOI: http://doi.org/10.1016/S00456535(99)00443-9.

(50) Vásquez A., Forsgren E., Fries I., Paxton RJ., Flaberg E., Szekely L. Symbionts as major modulators of insect health: lactic acid bacteria and honeybees. PLoS One. 2012; 7(3): e33188. DOI: http://doi. org/10.1371/journal.pone.0033188.

(51) Batchelder A. Chlortetracycline and oxytetracycline effects on plant growth and development in soil systems. J. Environ Qual. 1982; 11(4): 675-678. DOI: http://doi.org/10.2134/jeq1982.114675x.

(52) Wichmann F., Udikovic-Kolic N., Andrew S., Handelsman J. Diverse Antibiotic Resistance Genes in Dairy Cow Manure. mBio, 2014; 5(2): 5, e01017-13.

(53) Novais C., Freitas AR., Silveira E., Antunes P., Silva R., Coque TM. Spread of multidrug-resistant Enterococcus to animals and humans: an underestimated role for the pig farm environment. J. Antimicrob Chemother. 2013. DOI: http://doi.org/10.1093/jac/dkt289. 